\title{
Análisis numérico del ámbito de validez del modelo convencional de tiro oblicuo
}

\author{
Numerical analysis of the validity scope of conventional ballistiscs model
}

\author{
César Medina, Leonor Colombo de Cudmani
}

Facultad de Ciencias Exactas y Tecnología, Universidad Nacional de Tucumán, San Miguel de Tucumán, Argentina

Recebido em 14 de julho de 2015. Aceito em 8 de setembro de 2015

\begin{abstract}
En una encuesta de diagnóstico planteada a estudiantes del ciclo básico universitario sobre el tema de tiro oblicuo, se obtiene un alto porcentaje de respuestas incorrectas sobre estimaciones referidas al roce del aire y otros fenómenos que influyen en el desplazamiento de un proyectil. Estos desaciertos están asociados al hecho de que, tanto en los textos como en el aula, se emplea un modelo físico simplificado que supone que la velocidad sólo varía por la gravedad. Si bien esta simplificación es razonable en los primeros niveles de enseñanza, no se aclara debidamente que en muchos casos prácticos tal modelo idealizado puede generar errores sistemáticos significativos si no son debidamente analizados sus supuestos y ámbitos de validez. Como una estrategia didáctica para superar estas dificultades, se propone una comparación de las trayectorias del modelo convencional con otras calculadas numéricamente incluyendo distintas fuerzas y efectos (resistencia del medio, empuje, fuerza de Coriolis, perfil de densidad del aire). Las trayectorias se analizan bajo diferentes condiciones y se discute la factibilidad de algunas simplificaciones en términos del error sistemático que introducen.
\end{abstract}

Palabras-clave: tiro oblicuo, ámbito de validez, modelo convencional, análisis numérico, errores sistemáticos.

In a diagnosis survey about ballistics, given to a group of undergraduate students, a high percentage of wrong answers was obtained about estimates of air resistance and other phenomena affecting the movement of a projectile. These mistakes are related to a simplified model widely used in textbooks as well as in lectures. This model assumes that the variation of velocity depends only on gravity. Although this simplification is admissible at this level of teaching, it is not appropriately explained that in many practical cases this idealized model can lead to considerable systematic errors if the hypotheses and validity scope are not duly analyzed. A teaching strategy to overcome these difficulties is proposed. Trajectories of the conventional model are compared with other trajectories computed numerically, including different forces and effects (air resistance, buoyancy, Coriolis force, air density profile). The numerical trajectories are analyzed under different conditions. Also, the viability of some simplifications is discussed, considering the systematic error that they introduce.

Keywords: ballistics, validity scope, conventional model, numerical analysis, systematic errors.

\section{Introducción}

En el lenguaje común, suele asociarse los términos "tiro" o "balística" sólo con armas y proyectiles, pero el tema de tiro oblicuo se extiende a contextos mucho más amplios, asociados con importantes campos de la física tales como la mecánica de los fluidos. Entre sus aplicaciones más sencillas, las deportivas pueden ser fuente de motivación para los estudiantes. En este sentido, debe notarse que los conceptos y formalismos de este tema no sólo son útiles para actividades como el tiro al blanco o la caza, sino para todos los deportes en que interviene una pelota $u$ otro tipo de móvil, y en especial para las disciplinas olímpicas como el lanzamiento de disco, jabalina, bala, martillo, e incluso el salto.

*Endereço de correspondência: cesarmedina@arnet.com.ar 
La estrecha relación de este tema con el deporte y el juego no es casual. A partir de los primeros trabajos de Piaget, las teorías constructivistas del aprendizaje nos enseñan que desde el primer estadio evolutivo, el sensorio-motor (0-2 años de edad), el niño juega a arrojar objetos como una manera de explorar y afianzar su concepto del espacio y del movimiento, de medir su fuerza y mejorar su destreza. Además, si aceptamos que existe un paralelo entre la evolución del individuo y la de la especie humana, cabe considerar que en el período paleolítico, varios cientos de milenios antes de la invención de la rueda, homínidos anteriores al homo sapiens ya usaban armas arrojadizas. Estas consideraciones, además de poder ser usadas en el aula como motivación del aprendizaje, abonan el ordenamiento tradicional de este tema en los programas de física, donde suele ocupar el primer lugar entre los movimientos compuestos.

Desde una perspectiva didáctica, el tiro oblicuo permite un importante aprendizaje constructivo, exigiendo integración conceptual y destrezas operativas por parte de los estudiantes. Tanto en el nivel secundario como en la universidad, suele dictarse a continuación de movimiento rectilíneo y caída libre, y de hecho debe interpretarse como una clara oportunidad de síntesis de estos contenidos, pues no es otra cosa que la superposición o composición, en el plano, de esos dos movimientos en una sola dimensión.

En el enfoque didáctico tradicional de tiro oblicuo, se supone que la única fuerza es el peso, es decir, que la componente vertical de la velocidad sólo varía por gravedad, y la horizontal es constante. Se desprecia toda otra fuerza, como rozamiento, empuje, etc.

Si bien en estas primeras etapas de aprendizaje estas simplificaciones son razonables, es cuestionable que no suelan ser objeto de un análisis crítico por parte de los docentes ni de los textos. No se indica al alumno bajo qué condiciones y en qué medida son admisibles, y lo inducen a errores conceptuales que menoscaban su formación.

En este trabajo se presenta una encuesta, realizada en el ciclo básico universitario, que evidencia estas dificultades, y se propone una herramienta didáctica para superarlas. Se comparan las trayectorias del modelo convencional con otras calculadas numéricamente, asignando diferentes valores a los parámetros relevantes del fenómeno: ángulo de inclinación, velocidad inicial, y tamaño y densidad del proyectil. Se tiene en cuenta la fuerza de Coriolis y el empuje y resistencia del aire, asumiendo una variación exponencial de su densidad con la altitud.

\section{Encuesta sobre tiro oblicuo}

\subsection{Datos Generales}

Cantidad de formularios distribuidos: 57

Cantidad de formularios devueltos en blanco: 1

Al lado de cada opción se indica la cantidad de respuestas y el porcentaje, con precisión de dos dígitos, calculado sobre los formularios contestados (56).

\subsection{Preguntas:}

1. ¿Estudió el tema de tiro oblicuo?

- Sí, en la escuela secundaria y en la universidad: $27(48 \%)$

- Sí, en la escuela secundaria: $0(0 \%)$

- Sí, en la universidad: 29 (52\%)

- No estudié ese tema: $0(0 \%)$

2. ¿Le pareció un tema difícil?

- Sí, comparado con otros temas de física, me pareció difícil: 2 (3.6\%)

- Su grado de dificultad me pareció comparable al de otros temas: 25 (45\%)

- Me pareció fácil: 28 (50\%)

(Un alumno dejó este ítem sin responder)

3. ¿Le gustó el tema?

- Sí, me pareció interesante: 37 (66\%)

- No me despertó un interés especial: 19 $(34 \%)$

- No me gustó: 0 (0\%)

4. ¿Considera que es un tema importante en su formación profesional?

- Sí, me parece tanto o más importante que otros temas de física: 19 (34\%)

- Creo que otros temas de física fueron más importantes en mi formación: $32(57 \%)$

- Le encuentro muy poca importancia en mi formación: 3 (5.4\%)

(Un alumno dejó este ítem sin responder)

5. ¿Cree que este tema puede serle útil para algún interés personal, o mejorar su destreza en alguna actividad deportiva? (Ejemplos: tiro al 
blanco, lanzar una pelota con un dado efecto, darle mayor alcance, construir o diseñar un arma, etc.)

- Sí, tengo este tipo de intereses y creo que este tema puede serme útil: $20(36 \%)$

- Tengo este tipo de intereses, pero no creo que este tema pueda serme útil: $2(3.6 \%)$

- Si tuviera ese tipo de intereses, creo que podría serme útil: $34(61 \%)$

- Aunque tuviera ese tipo de intereses, no creo que pudiera serme útil: $0(0 \%)$

6. Considere el disparo de proyectiles de plomo de distintos calibres o diámetros $(5 \mathrm{~mm}, 10$ $\mathrm{mm}, 30 \mathrm{~mm}$ ). Los disparos se realizan en un ángulo de $45^{\circ}$ respecto de la horizontal y a una velocidad de $100 \mathrm{~m} \cdot \mathrm{s}^{-1}$ (puede pedir al docente otros datos que considere necesarios). Califique de verdadera o falsa a cada una de las siguientes proposiciones: En todos o la mayoría de estos casos, a los fines de calcular el alcance horizontal del tiro, con un error del $10 \%$, se pueden despreciar los siguientes parámetros o fenómenos:

a) La resistencia del aire al desplazamiento del proyectil.

Verdadera: 21 (37\%) Falsa: $24(43 \%)^{*}$

No sé: 10 (18\%)

b) La viscosidad del aire.

Verdadera: $31(55 \%)^{*}$ Falsa: $11(20 \%)$

No sé: $13(23 \%)$

c) El empuje del aire (principio de Arquímedes).

Verdadera: $42(75 \%) *$ Falsa: $8(14 \%)$

No sé: 5 (8.9\%)

d) El movimiento de rotación de la Tierra.

Verdadera: $40(71 \%)^{*}$ Falsa: $9(16 \%)$

No sé: $6(11 \%)$

e) La variación de la densidad del aire con la altitud.

Verdadera: $37(66 \%) *$ Falsa: $8(14 \%)$

No sé: 10 (18\%)

(Un alumno dejó este ítem sin responder.

Las respuestas correctas están señaladas con un asterisco).

7. Puede, si lo desea, hacer comentarios u observaciones. Gracias por su colaboración.

- Creo que tomábamos despreciables estos fenómenos en casos así.
- En las clases de física no recuerdo haber visto casos que consideraran estas variables. Recuerdo haber analizado casos ideales.

\subsection{Descripción y análisis de los resultados de cada ítem}

1. Todos los alumnos estudiaron el tema en la universidad, y casi la mitad de ellos lo estudió también en la escuela secundaria.

2. A la mitad de los alumnos le pareció un tema fácil, y sólo al $3.6 \%$ le pareció difícil.

3. A dos de cada tres alumnos les pareció un tema interesante, y al resto no le despertó un interés especial, pero ninguno contesta que no le haya gustado.

4. Más de la mitad de los alumnos considera que otros temas de física fueron más importantes en su formación, y sólo un tercio considera que este tema es tanto o más importante que otros temas. Unos pocos $(5.4 \%)$ le encuentran muy poca importancia.

5. Más de un tercio de los alumnos declaran tener intereses lúdicos o deportivos asociados al tiro oblicuo y consideran que este tema de estudio puede servirles a tales fines. Unos pocos $(3.6 \%$, equivalente a la décima parte de los anteriores) declaran tener este tipo de intereses pero no creen que este tema pueda servirles. El resto $(61 \%)$, una clara mayoría, no tiene este tipo de intereses, pero considera que este tema podría servirle si los tuviera.

6. El primer ítem es el que tiene menos aciertos: sólo el $43 \%$ de los alumnos, es decir, una minoría, sabe o intuye que no puede despreciarse el efecto de la resistencia del aire en el desplazamiento del proyectil. Los que están equivocados al respecto $(37 \%)$ son más del doble de los que simplemente lo ignoran (18\%).

En los demás ítems, la mayoría acierta en sus respuestas pero nuevamente la mayor cantidad de desaciertos e ignorancia se observa respecto de la viscosidad, asociada a la resistencia del aire, más precisamente al roce propiamente dicho, es decir, a las fuerzas de fricción tangenciales a la superficie del proyectil. Cabe destacar que tanto la fuerza de roce en general, como la viscosidad en particular, son temas estudiados en la misma asignatura aunque en forma separada de tiro oblicuo. 
Por el contrario, con respecto al principio de Arquímedes, que también es estudiado tanto en la escuela secundaria como en la universidad, se observa la mayor cantidad de aciertos (ya sea por intuición o conocimiento) y el menor porcentaje de ignorancia.

En cuanto al efecto de la rotación de la Tierra, hay también un alto porcentaje de aciertos que suponemos asociado a la intuición, dado que los programas del ciclo básico suelen dedicar menos tiempo a este tema que a la mecánica de los fluidos.

Dos alumnos (3.6\%) comentan que, según recuerdan, consideraron despreciables los fenómenos mencionados, o bien se omitió su consideración.

\subsection{Conclusiones de la encuesta}

La mitad de los alumnos considera que tiro oblicuo es un tema fácil, y a dos tercios les parece interesante, pero sólo a un tercio le parece tanto o más importante que otros temas para su formación. Es claro que los alumnos distinguen claramente entre facilidad y gusto, por un lado, e importancia o valoración, por otro. Las respuestas a la pregunta 5 sugieren que la facilidad y el gusto pueden estar asociados, al menos en parte, a cuestiones lúdicas (pasatiempos, deportes, etc.).

Creemos que es inmerecida la falta de valoración, por parte de los alumnos, sobre la capacidad formativa de este tema. Al parecer ellos no advierten que sus aspectos formales, metodológicos y operativos suponen un importante aprendizaje constructivo. En particular, su sencillez, su claridad fenomenológica y su arraigo cognitivo lo hacen especialmente apto para introducir el principio de composición de movimientos independientes, que se aplica a temas más complejos de mecánica y da la base formal para abordar otros contenidos de física y matemática. Si se destacaran estos aspectos durante el estudio de este tema, probablemente los estudiantes valorarían más su capacidad formativa y se sentirían más motivados.

La mayoría de los alumnos evidencia confusión o ignorancia respecto del importante papel que desempeña la resistencia del aire en estos casos prácticos de tiro oblicuo.

\section{Propuesta didáctica}

\subsection{Análisis físico}

Para obtener la trayectoria de un proyectil que se desplaza por el aire, planteamos su ecuación de movimiento ( $2^{a}$ ley de Newton)

$$
\mathbf{F}=m \cdot \mathbf{a},
$$

donde la fuerza es la suma del peso $\mathbf{P}$, el empuje $\mathbf{E}$ (debido al principio de Arquímedes), la resistencia del aire $\mathbf{F}_{r}$, y la fuerza de Coriolis $\mathbf{F}_{c}$

$$
\mathbf{P}+\mathbf{E}+\mathbf{F}_{\mathbf{r}}+\mathbf{F}_{\mathbf{c}}=m \frac{\mathrm{d} \mathbf{v}}{\mathrm{d} t},
$$

siendo $\mathbf{v}$ la velocidad.

En la Ec. (2), $\mathbf{P}$ y $\mathbf{E}$ tienen signos opuestos, negativo y positivo, respectivamente, pues el peso está dirigido hacia abajo y el empuje hacia arriba. La resistencia del aire, por su parte, tendrá siempre signo opuesto al de la velocidad. Esta fuerza también es llamada, en sentido lato, "rozamiento" pero, como se verá más adelante, sólo una parte de ella corresponde a la fricción o rozamiento propiamente dicho. Está dada por (Calderón et al. [1])

$$
\mathbf{F}_{\mathbf{r}}=-\operatorname{sgn}(\mathbf{v}) \cdot \frac{1}{2} C_{p} \rho_{a} A \mathbf{v}^{2}
$$

donde $-\operatorname{sgn}(\mathbf{v})$ da cuenta del signo opuesto a la velocidad, y se incluye el factor 0.5 para destacar que el trabajo que realiza esta fuerza es proporcional a la energía cinética transferida por el proyectil al aire. $\rho_{a}$ es la densidad del medio, $A$, el área transversal del cuerpo con respecto a la velocidad, y $C_{p}$, el coeficiente de arrastre o de presión, que para el caso de móviles esféricos -a los cuales se limitará este estudio- puede calcularse como

$$
C_{p}=\frac{24}{R e}+\frac{1}{1+\sqrt{R e}}+0,4
$$

En la Ec. (4), Re es el número de Reynolds, dado por

$$
R e=\frac{\rho_{a} \cdot d \cdot v}{\eta}
$$

donde $\eta$ representa la viscosidad del medio, y $d$ es un parámetro de longitud característico del móvil, que en nuestro caso será el diámetro del proyectil esférico.

El primer término de la Ec. (4) corresponde a la fricción o rozamiento propiamente dicho, es decir, a las fuerzas tangenciales sobre el móvil, las cuales predominan en régimen laminar, a bajas velocidades. $\mathrm{El}$ segundo es un término de ajuste empírico que da cuenta de las transiciones entre distintos regímenes; y el tercero es el valor del coeficiente aerodinámico de una esfera, representativo del intervalo $R e<2 \times 10^{5}$. 
Este último término está asociado a las fuerzas de presión, también llamadas de arrastre o inerciales (perpendiculares a la superficie del cuerpo), es decir, al intercambio de momento entre el cuerpo y las moléculas del fluido, predominante en régimen turbulento, a altas velocidades.

Además del número de Reynolds, cuyo valor define el tipo de régimen (laminar o turbulento) es necesario considerar otro número adimensional, también dependiente de la velocidad: el número de Mach, $M$, que es simplemente el cociente entre la velocidad del móvil y la del sonido en el medio que se trate. En aire a $20{ }^{\circ} \mathrm{C}$ de temperatura, la velocidad del sonido es $v_{s}=343 \mathrm{~m} \cdot \mathrm{s}^{-1}$.

La Ec. (4) es válida para $M \leqslant 0,3$, rango en el cual puede considerarse que el aire se comporta como un fluido incompresible; en tanto que para $0,3<M<0,7$, debe hacerse una corrección a $C_{p}$, debido a la compresibilidad del aire. Según la analogía de Prandtl-Grauer (Roncero y Jiménez [2])

$$
C_{p}=\frac{C_{p, i n c}}{\sqrt{1-M^{2}}}
$$

donde el primer miembro representa el valor del coeficiente de arrastre corregido por compresibilidad, y $C_{p, i n c}$ su valor para el caso de fluido incompresible. Para $M>0,7$, la Ec. (6) deja de ser válida $\left(C_{p}\right.$ no puede tender a infinito) y comienza el régimen transónico, cuyas complejidades están fuera del contexto de este trabajo. La velocidad del sonido depende de la temperatura, y por tanto, de la altura; pero para nuestra temperatura de referencia y los rangos de altura de nuestros cálculos, la variación es del orden del $0.4 \%$, y a los fines de aplicar la Ec. (6), que es un factor de corrección, esta variación es despreciable.

La fuerza de Coriolis, por su parte, da cuenta de la variación de la velocidad asociada a la rotación de la Tierra, y está dada por

$$
\mathbf{F}_{\mathbf{c}}=2 m \boldsymbol{\omega} \times \mathbf{v}
$$

donde $\boldsymbol{\omega}$ es la velocidad angular de la Tierra.

Esta fuerza depende de la latitud. Por simplicidad, en nuestro estudio bidimensional consideraremos un plano vertical a lo largo del ecuador, donde la velocidad puede tener sólo componentes este-oeste $(x)$ y vertical $(z)$ que se afectan recíprocamente con la máxima intensidad, es decir, a $0^{\circ}$ lat. la Ec. (7) predice el máximo efecto de $v_{x}$ en $v_{z}$ y viceversa.

\subsection{Análisis numérico}

Reemplazando las Ecs. (3) y (7) en la (2), obtenemos una ecuación diferencial para la velocidad como función del tiempo. Antes de resolverla, conviene hacer algunas simplificaciones algebraicas, teniendo en cuenta que peso $=$ gravedad $\cdot$ masa, y masa $=$ densidad $\cdot$ volumen. Así, en diferencias finitas se tendrá

$$
\Delta \mathbf{v}=\left[-\mathbf{g}\left(1-\frac{\rho_{a}}{\rho_{p}}\right)-3 / 8 \frac{C_{p} \rho_{a}}{r \rho_{p}} \mathbf{v}^{2}+2 \boldsymbol{\omega} \times \mathbf{v}\right] \Delta t
$$

donde $\boldsymbol{\omega}=7,27 \times 10^{-5} \mathrm{~s}^{-1}$ y $\rho_{p}$ es la densidad del proyectil. Esta ecuación es importante para interpretar algunos de nuestros resultados: al dividir la fuerza en la masa para obtener la aceleración, se obtiene que, "aun cuando la resistencia del aire es proporcional al área $A$ transversal del proyectil, es decir al cuadrado del radio (Ec. (3)), la deceleración que esta fuerza provoca es inversamente proporcional al radio del proyectil (segundo término del segundo miembro de la Ec. (8))". Esta idea deberá tenerse presente al analizar cómo varían las trayectorias con el radio del proyectil.

Para la densidad del aire $\rho_{a}$ se supone un perfil dado por (Rishbeth and Garriot, [3])

$$
\rho_{a}=\rho_{a o} \mathrm{e}^{-h / H} .
$$

Siendo $\rho_{a o}$ el valor de la densidad del aire a nivel del mar $\left(\right.$ a $\left.20{ }^{\circ} \mathrm{C}, \rho_{a o}=1,2 \mathrm{~kg} \cdot \mathrm{m}^{-3}\right) ; h$, la altitud, y $H$, la altura de escala (o longitud de escala de gradiente de densidad) dada por

$$
H=\frac{R T}{M g},
$$

donde $R$ es la constante universal de los gases $\left(R=8,31 \mathrm{~N} \cdot \mathrm{m} \cdot \mathrm{mol}^{-1} \cdot \mathrm{K}^{-1}\right) ; T$, la temperatura absoluta (en nuestro caso, $T=293.15 \mathrm{~K},\left[20{ }^{\circ} \mathrm{C}\right]$ ); $M$, la masa molar del aire $\left(M=0,0289644 \mathrm{~kg} \cdot \mathrm{mol}^{-1}\right)$ y $g$ la gravedad $\left(9,8 \mathrm{~m} \cdot \mathrm{s}^{-2}\right)$. Luego, en este contexto, la altura de escala es $H=8582 \mathrm{~m}$.

La gravedad, la temperatura y la viscosidad del aire se consideran constantes a cualquier altura (la viscosidad en los gases no varía apreciablemente con la densidad, $\left.\eta=1,8 \times 10^{-5} \mathrm{~N} \cdot \mathrm{s} \cdot \mathrm{m}^{-2}\right)$.

A fin de calcular las trayectorias se resuelve la Ec. (8) usando el método de Runge-Kutta de $5^{\circ}$ 
orden, con paso de tiempo adaptativo regulado por control PID (Proporcional-Integral-Derivativo), imponiendo una tolerancia local (es decir, en cada paso de tiempo) relativa $\varepsilon=5 \times 10^{-4}$, más una tolerancia local absoluta $\Delta v=1 \times 10^{-4} \mathrm{~m} \cdot \mathrm{s}^{-1}$.

Para las coordenadas vertical y horizontal se aplica un método de primer orden, dado que en cada paso de tiempo la velocidad media puede considerarse la obtenida por el método de quinto orden.

Se estudia cómo varían las trayectorias cuando se asignan diferentes valores a un dado conjunto de parámetros, a saber: el ángulo de inclinación $(\alpha)$, la velocidad inicial $\left(v_{o}\right)$, y el radio y la densidad del proyectil $\left(r\right.$ y $\left.\rho_{p}\right)$. Los parámetros se varían de a uno por vez, asignándoles tres valores arbitrarios, uno de los cuales se toma como referencia. El conjunto de valores de referencia es: $\alpha=45^{\circ}, v_{o}=70 \mathrm{~m} \cdot \mathrm{s}^{-1}$, $r=1 \mathrm{~cm} \mathrm{y} \rho_{p}=4 \mathrm{~g} \cdot \mathrm{cm}^{-3}$.

La elección de los valores obedece a razones prácticas. En todos los parámetros, se ha intentado abarcar un rango amplio sin llegar a extremos triviales o atípicos. Para el ángulo de inclinación se ha tomado $45^{\circ}$ como referencia porque este valor corresponde, en el modelo convencional, al máximo alcance horizontal, que es la magnitud que se toma como base de comparación entre las trayectorias, como se verá más adelante. Los valores de la velocidad, el radio y la densidad del proyectil se duplican de un caso a otro. Así, por ejemplo, la máxima velocidad considerada es el doble de la de referencia, y ésta es el doble de la mínima. El límite superior de este parámetro está condicionado por el régimen subsónico; se ha impuesto que la variación del coeficiente $C_{p}$ producida por la Ec. (6) no supere un $10 \%$.

La Tabla 1 muestra los distintos valores considerados para cada parámetro, y el conjunto de referencia sobre fondo gris.

Finalmente, se resuelve la Ec. (8) para algunos casos particulares, a fin de evaluar la contribución relativa de distintas fuerzas, y el efecto del perfil de la Ec. (9).

Tabla 1: Valores de los parámetros de tiro oblicuo.

\begin{tabular}{lccc}
\hline$\alpha\left(^{\circ}\right)$ & $\mathrm{v}_{o}\left(\mathrm{~ms}^{-1}\right)$ & $r(\mathrm{~cm})$ & $\rho_{p}\left(\mathrm{gcm}^{-3}\right)$ \\
\hline 5 & 35 & 0.5 & 2 \\
45 & 70 & 1 & 4 \\
85 & 140 & 2 & 8 \\
\hline
\end{tabular}

\section{Resultados}

\subsection{Efectos de los parámetros del tiro}

La Fig. 1 compara las trayectorias obtenidas para tres ángulos de inclinación: $\alpha=5^{\circ}$ (arriba), $\alpha=45^{\circ}$ (centro), $\alpha=85^{\circ}$ (abajo). En todos los casos, los valores de los demás parámetros son los de referencia (fila central de la Tabla 1). Las curvas "a" muestran las trayectorias numéricas, que incluyen empuje, fuerza de Coriolis y resistencia del aire. La curva "b" (la misma en los tres recuadros) corresponde a la parábola del modelo convencional. Los segmentos rectos, entre ambas curvas, unen puntos de ellas que corresponden a un mismo instante, y están espaciados a intervalos de 1 segundo. Nótese que, por razones de claridad, las escalas de los ejes vertical y horizontal son distintas en cada caso (en cada recuadro), y aun dentro de un mismo caso, son distintas en cada eje. De hecho, no se desea visualizar una comparación cuantitativa del efecto de variar los parámetros, sino una comparación cualitativa de dos tipos de trayectoria, bajo distintas condiciones.

La menor diferencia entre el modelo numérico y el convencional se observa para el ángulo de inclinación más rasante $\left(\alpha=5^{\circ}\right)$. Para $\alpha=45^{\circ}$ se tiene la mayor diferencia en alcance horizontal, y para $\alpha=85^{\circ}$ se tiene la mayor diferencia en alcance vertical. Las trayectorias numéricas no son simétricas: para $\alpha=45^{\circ}$, la rama descendente tiene una pendiente más abrupta que la ascendente, en tanto que para $\alpha=85^{\circ}$ sucede lo contrario. Los segmentos rectos muestran que en todos los casos las trayectorias numéricas tienen una duración menor que la convencional. Para cualquier instante, la altura en la trayectoria numérica es igual o menor que en la parábola convencional.

La Fig. 2 muestra las trayectorias para distintos valores de velocidad inicial, que se duplica de un caso a otro: $v_{o}=35 \mathrm{~m} \cdot \mathrm{s}^{-1}$ (arriba), $v_{o}=70 \mathrm{~m} \cdot \mathrm{s}^{-1}$ (centro), y $v_{o}=140 \mathrm{~m} \cdot \mathrm{s}^{-1}$ (abajo, donde se usó la Ec. (6) para corregir el valor de $C_{p}$, debido a que $M>0,3)$. Desde luego, cuanto mayor es la velocidad inicial, mayor es el alcance horizontal y vertical, en términos absolutos. Sin embargo, en términos comparativos con respecto a la trayectoria convencional, se observa que a medida que aumenta la velocidad inicial, las trayectorias numéricas tienen un alcance vertical y horizontal menor, como cabe esperar de la dependencia de la fuerza de roce con la velocidad. 


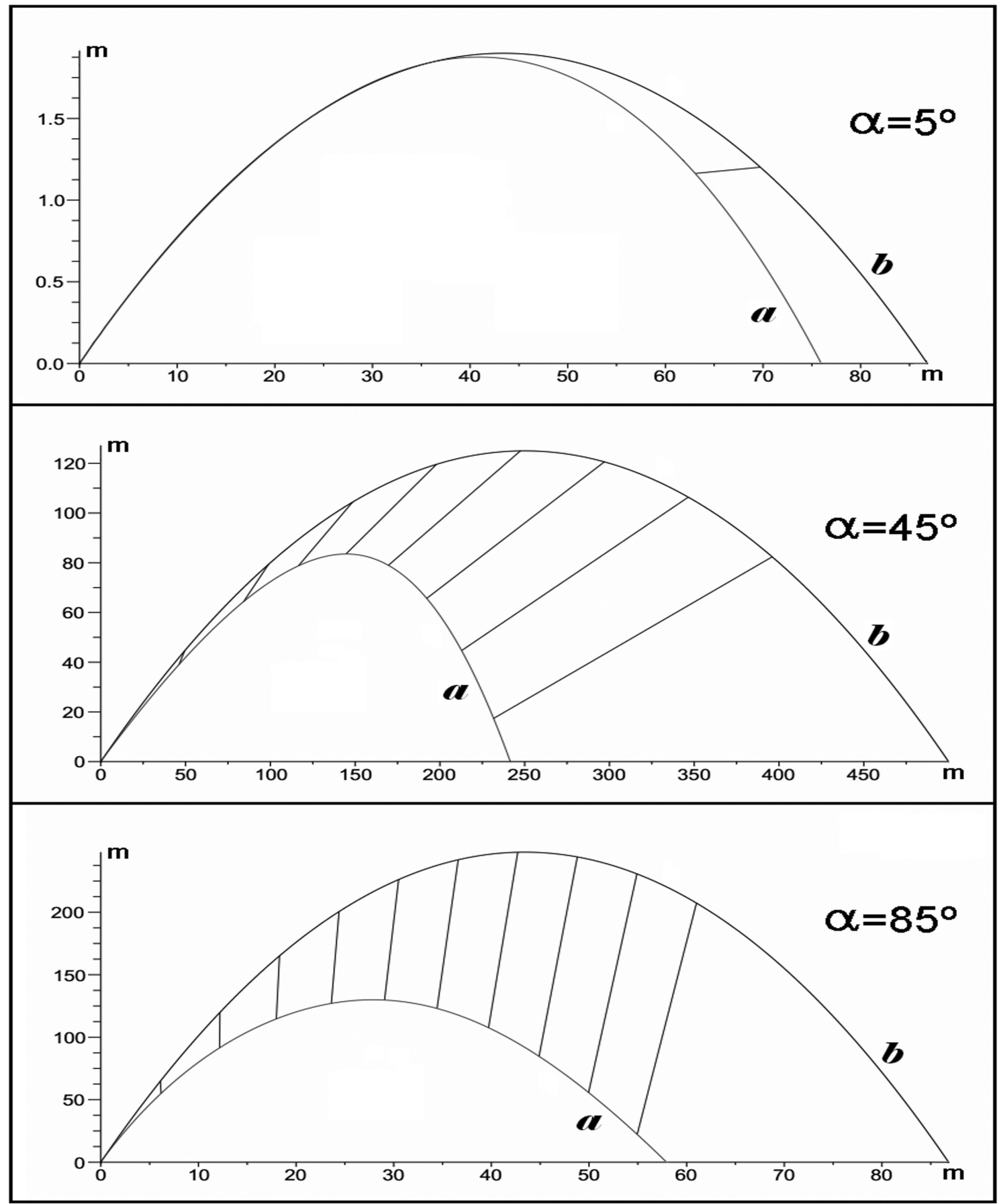

Figura 1: Comparación entre trayectorias numéricas (a) y convencional (b) para tres ángulos de inclinación: $\alpha=5^{\circ}$ (arriba), $\alpha=45^{\circ}$ (centro), $\alpha=85^{\circ}$ (abajo) (los segmentos rectos unen puntos correspondientes a un mismo instante).

Para $v_{o}=140 \mathrm{~m} \cdot \mathrm{s}^{-1}$ (abajo), tanto el alcance vertical como el horizontal están notablemente reducidos (aproximadamente una tercera y una quinta parte de la parábola convencional, respectivamente). En este caso, los segmentos rectos muestran claramente que el tiempo de vuelo guarda relación con la longitud de la trayectoria: en el mismo instante que, de acuerdo a la curva numérica, el proyectil ya ha caído al suelo, en la parábola convencional está a más de $400 \mathrm{~m}$ de altura (apenas ha iniciado el descenso).

La Fig. 3 compara las trayectorias para tres distintos valores del radio del proyectil, que se duplica de un caso a otro: a) $r=0.5 \mathrm{~cm}, \mathrm{~b}) r=1 \mathrm{~cm}$, c) $r=2 \mathrm{~cm}$, y la trayectoria convencional d). Nótese que la diferencia de tamaño de las parábolas 


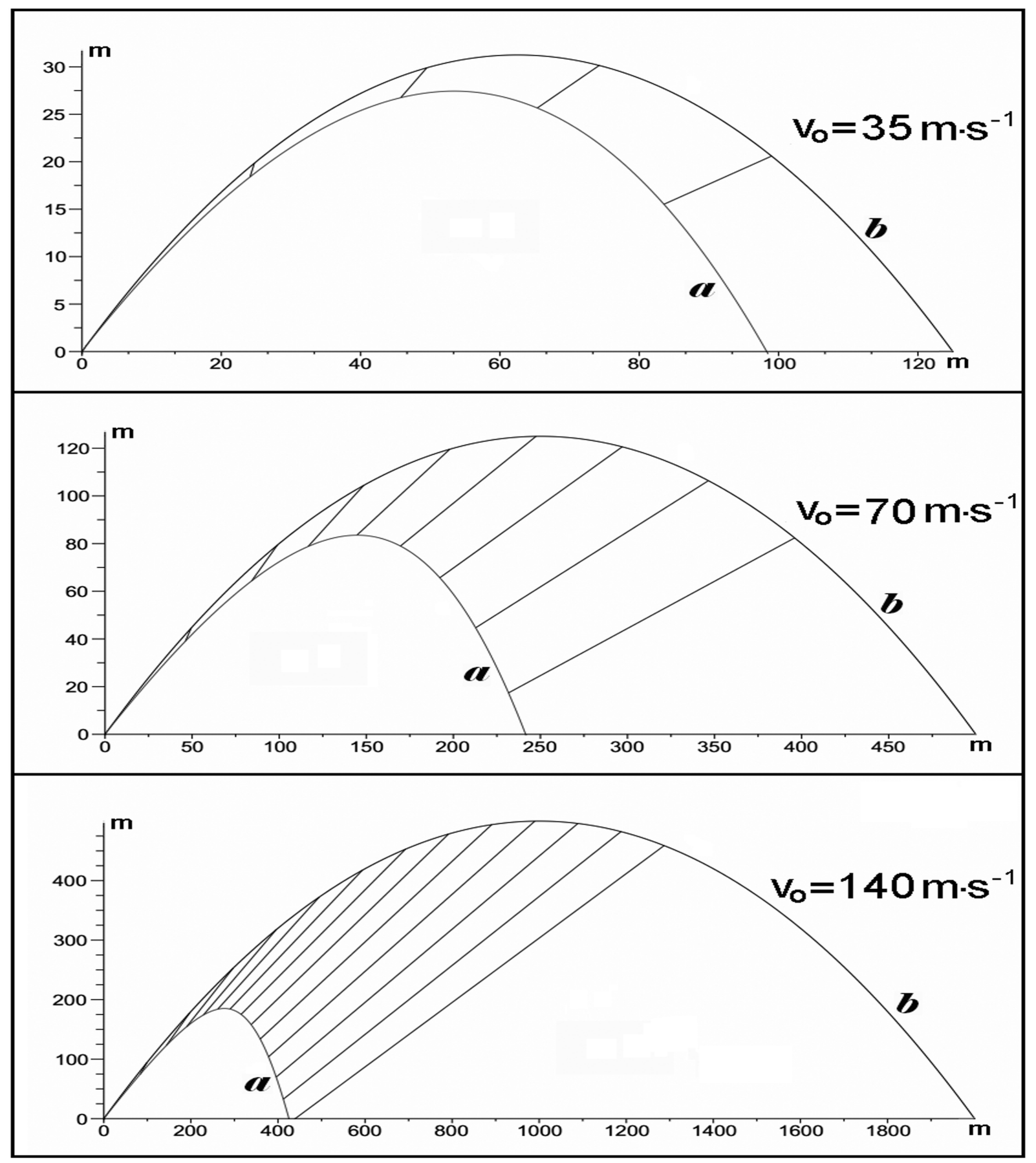

Figura 2: Ídem a Fig. 1 pero para tres velocidades iniciales: $v_{o}=35 \mathrm{~m} \cdot \mathrm{s}^{-1}$ (arriba), $v_{o}=70 \mathrm{~m} \cdot \mathrm{s}^{-1}$ (centro), y $v_{o}=140 \mathrm{~m} \cdot \mathrm{s}^{-1}$ (abajo).

numéricas es algo mayor entre a) y b) que entre b) y c), a pesar de que la progresión es la misma en términos relativos del radio, y en términos absolutos es incluso menor entre a) y b). A medida que el tamaño del proyectil aumenta, las trayectorias numéricas se aproximan a la convencional; es decir, la resistencia o roce del aire, en términos relativos, es menos efectiva. Esto se explica porque la deceleración que provoca esta fuerza es inversamente proporcional al radio, como ya se adelantó a propósito de la Ec. (8). Este resultado lleva a una conclusión bastante intuitiva: si se disparan dos proyectiles de distinto tamaño con la misma velocidad inicial, el roce frena más al más pequeño.

La Fig. 4 compara las trayectorias para tres distintos valores de la densidad del proyectil, que se 


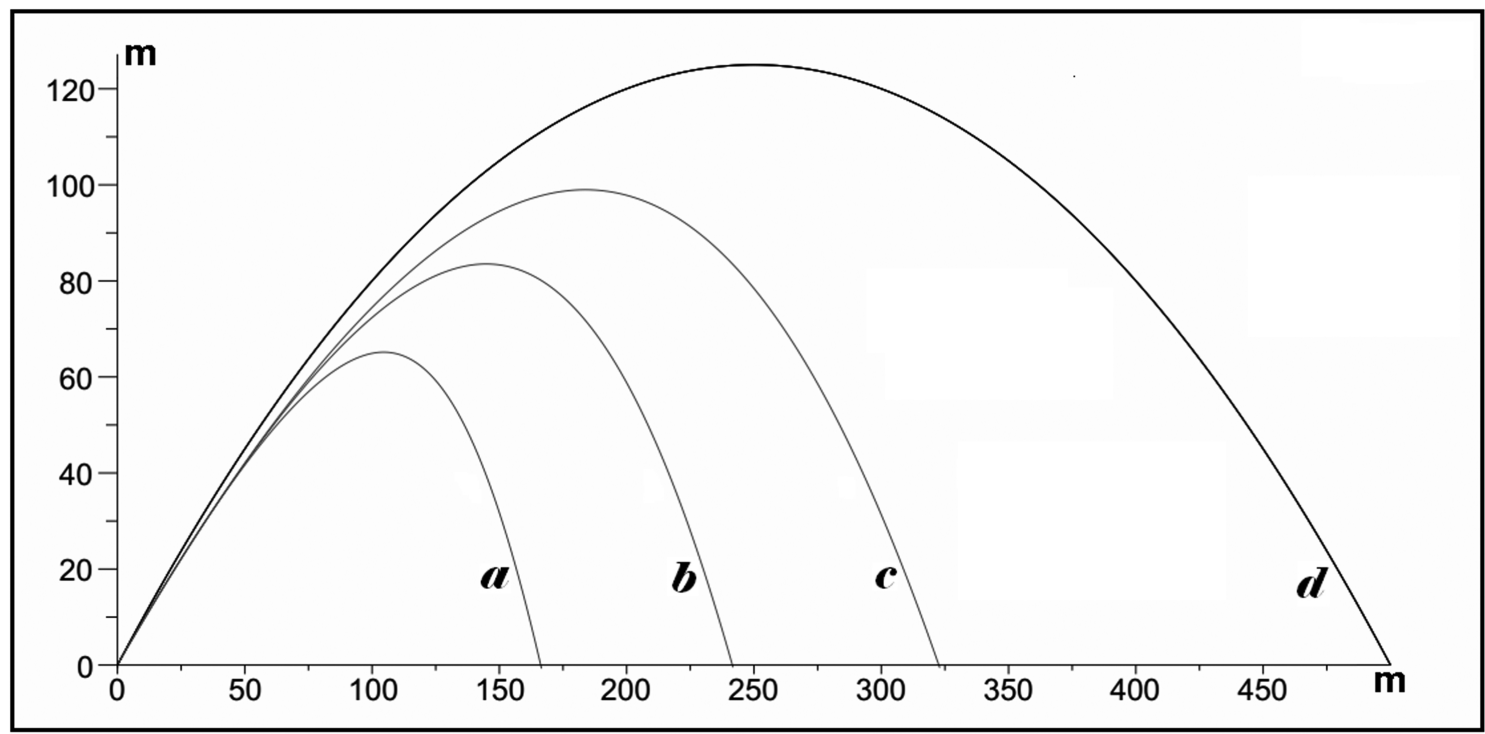

Figura 3: Comparación entre trayectorias numéricas para tres radios del proyectil: a) $r=0.5 \mathrm{~cm}, \mathrm{~b}) r=1 \mathrm{~cm}, \mathrm{c}) r=2$ $\mathrm{cm}$, y la trayectoria convencional $\mathrm{d}$ ) independiente del radio.

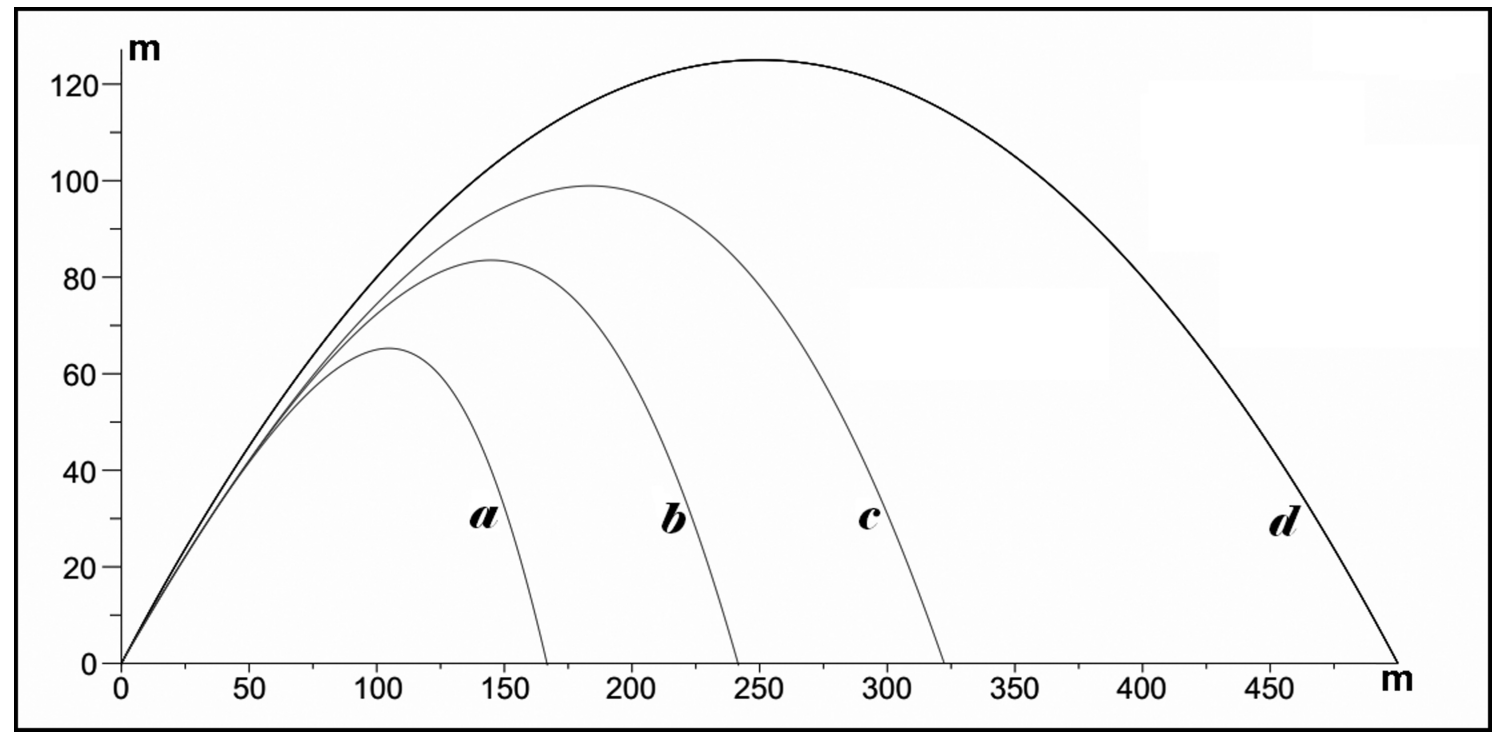

Figura 4: Ídem a Fig. 3 pero para tres densidades del proyectil: a) $\left.\left.\rho_{p}=2 \mathrm{~g} \cdot \mathrm{cm}^{-3}, \mathrm{~b}\right) \rho_{p}=4 \mathrm{~g} \cdot \mathrm{cm}-3, \mathrm{y} \mathrm{c}\right) \rho_{p}=8 \mathrm{~g} \cdot \mathrm{cm}-3$.

duplica de un caso a otro: a) $\rho_{p}=2 \mathrm{~g} \cdot \mathrm{cm}^{-3}$, b) $\rho_{p}=4 \mathrm{~g} \cdot \mathrm{cm}^{-3}$, y c) $\rho_{p}=8 \mathrm{~g} \cdot \mathrm{cm}^{-3}$, y la trayectoria convencional $\mathrm{d}$ ). Se observa que cuando la densidad del proyectil aumenta, las trayectorias numéricas se aproximan a la convencional; es decir, el efecto del roce, en términos relativos, es menor. Nótese que esta figura es igual a la Fig. 3, al menos dentro del nivel de resolución de ambas. Esto se debe a que, en este caso, los valores de densidad varían en la misma progresión que los del radio en el caso anterior, a partir de un mismo conjunto de referencia, y al hecho de que la resistencia del aire (Ec. (8)) depende exactamente igual del radio que de la densidad del proyectil. Esto se analizará con mayor detalle en la siguiente sección.

\subsection{Contribución relativa de los distintos términos de fuerza}

A fin de calcular en qué medida las trayectorias se ven afectadas por las distintas componentes de la fuerza, tomaremos como magnitud de referencia el alcance horizontal del tiro, cuya variación es el efecto más notorio para ángulos de inclinación $\alpha \leqslant 45^{\circ}$. 
En cuanto al empuje, en todos los casos estudiados, el hecho de despreciarlo introduce entre $0.1 \mathrm{y}$ $0.2 \%$ de variación en el alcance horizontal, lo cual puede verificarse en el cálculo numérico, pero no se aprecia en las gráficas, al menos en su nivel de resolución.

Lo mismo ocurre con la componente de fricción de la resistencia del aire, que produce variaciones aun menores ( $\sim 0.03 \%$, lo cual es comparable a la tolerancia local del cálculo). Nótese que la fricción, a pesar de ser inversamente proporcional al cuadrado del radio (a través de $R e$, que es proporcional al diámetro, v. Ecs. [3-5, 8]) no introduce ninguna diferencia entre las curvas de las Figs. 3 y 4, es decir, es completamente despreciable dentro del nivel de resolución de las gráficas. Esto se debe al elevado valor del número de Reynolds, $R e=O\left(10^{5}\right)$, que corresponde en todos los casos a franca turbulencia (Feynman et al. [4]).

En realidad, las fuerzas inerciales comienzan a superar a las viscosas mucho antes de que el flujo sea efectivamente turbulento: a partir de $R e=60$ (lo cual puede comprobarse igualando el primer término con el último, en el segundo miembro de la Ec. (4)). En el caso de una esfera de $1 \mathrm{~cm}$ de radio (nuestro valor de referencia) desplazándose en el aire, este valor de $R e$ corresponde a una velocidad $v=9 \mathrm{~cm}$. $\mathrm{s}^{-1}$, valor inviable para una velocidad inicial, pues en cualquier caso práctico implicaría esencialmente una caída libre.
Con respecto a la fuerza de Coriolis, su efecto en el alcance horizontal es del orden del $0.02 \%$ en la mayoría de los casos estudiados, y no puede apreciarse en las gráficas; pero es más notorio para proyectiles que alcanzan grandes alturas. La Fig. 5 muestra una trayectoria que considera la fuerza de Coriolis (a) y otra que la desprecia (b). Ambas se calcularon con el siguiente conjunto de parámetros: $\alpha=85^{\circ}, v_{o}=150 \mathrm{~m} \cdot \mathrm{s}^{-1}, r=1 \mathrm{~cm}$, y $\rho_{p}=11 \mathrm{~g}$. $\mathrm{cm}^{-3}$. Se observa que la fuerza de Coriolis reduce el alcance horizontal en un $0.6 \%$.

\subsection{Efecto del perfil de densidad del aire}

El cálculo del roce, a lo largo de la trayectoria, es más preciso si se tiene en cuenta que la densidad del aire disminuye al aumentar la altitud. Si para el caso de referencia, en lugar de suponer un valor constante de densidad $\rho_{a o}$ (valor a nivel del mar), se usa el perfil de la Ec. (9), se obtiene un alcance horizontal un $0.4 \%$ mayor.

Obviamente, este tipo de diferencia será más notorio para proyectiles que alcancen grandes alturas. La Fig. 6 muestra una trayectoria calculada con densidad constante y otra con la Ec. (9), con el siguiente conjunto de parámetros: $\alpha=60^{\circ}, v_{o}=150 \mathrm{~m} \cdot \mathrm{s}^{-1}$, $r=1 \mathrm{~cm}$, y $\rho_{p}=11 \mathrm{~g} \cdot \mathrm{cm}^{-3}$. El alcance horizontal con la densidad constante es un $1.4 \%$ menor.

(Estos resultados concuerdan con lo señalado por Perelman [5] en su obra de divulgación Física Recreativa I, donde comenta que este hecho fue descubierto en forma casual por los artilleros alemanes en la Pri-

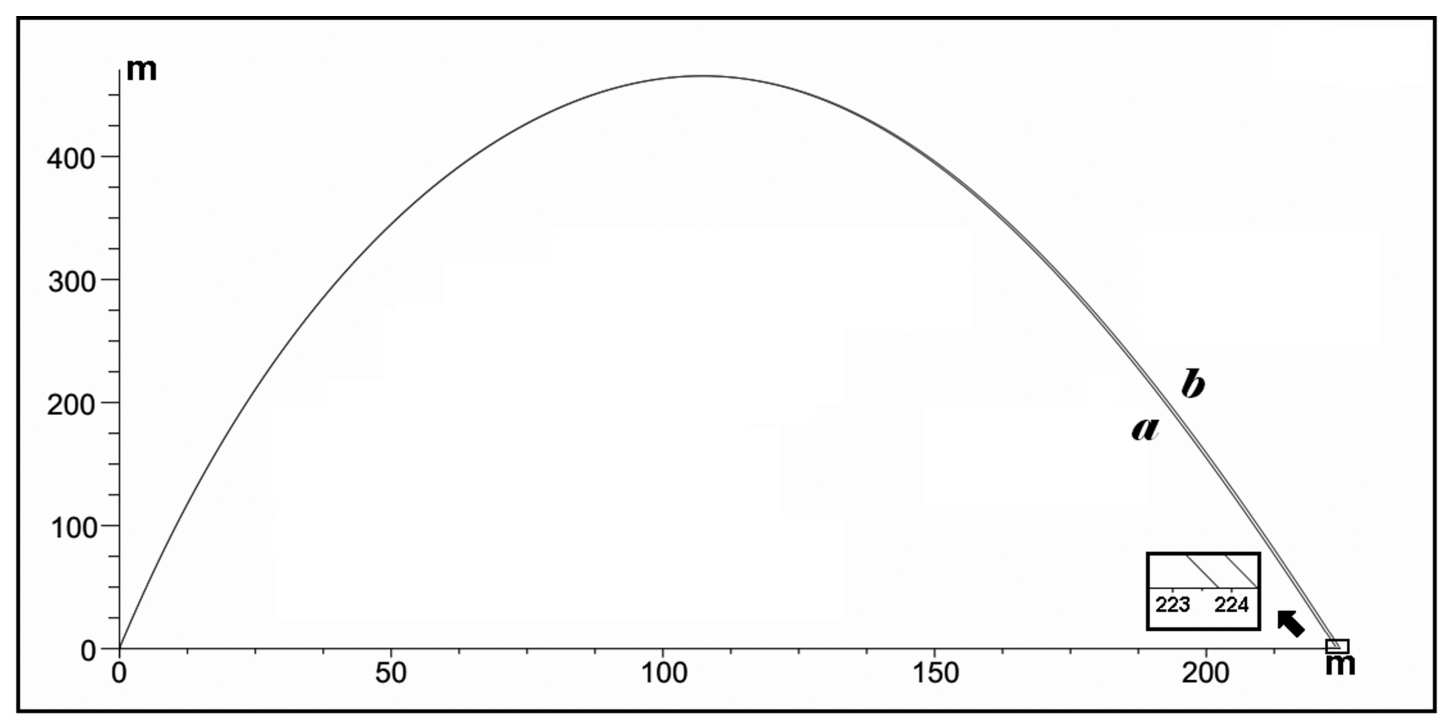

Figura 5: Comparación entre trayectorias numéricas considerando la fuerza de Coriolis (a) y despreciándola (b) (conjunto de parámetros $\alpha=85^{\circ}, v_{o}=150 \mathrm{~m} \cdot \mathrm{s}^{-1}, r=1 \mathrm{~cm}$, y $\left.\rho_{p}=11 \mathrm{~g} \cdot \mathrm{cm}^{-3}\right)$. 


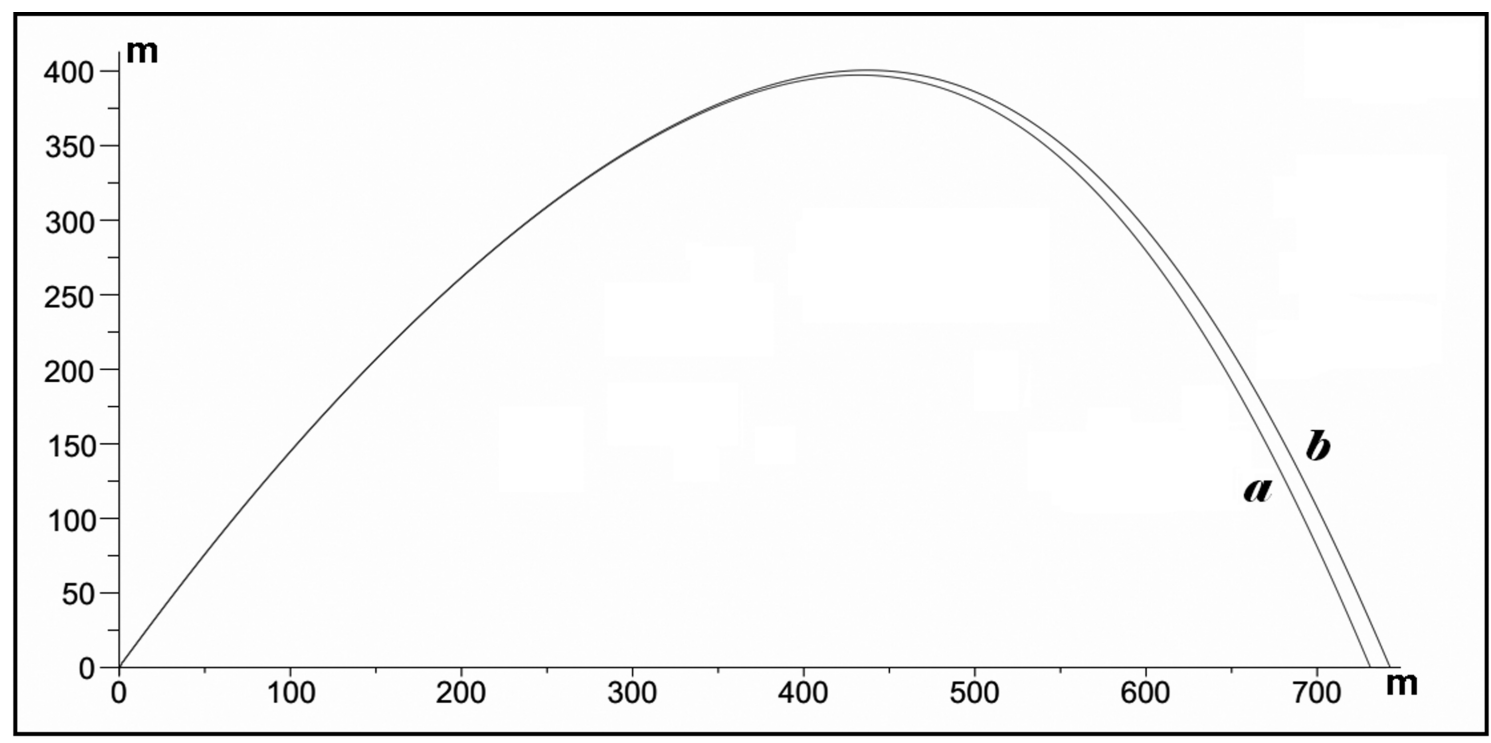

Figura 6: Comparación entre trayectorias numéricas considerando densidad del aire constante (valor al nivel del mar) a toda altitud (a), y el perfil de la Ec. (9) (b) (conjunto de parámetros $\alpha=60^{\circ}, v_{o}=150 \mathrm{~m} \cdot \mathrm{s}^{-1}, r=1 \mathrm{~cm}, \mathrm{y} \rho_{p}=11 \mathrm{~g} \cdot \mathrm{cm}{ }^{-3}$ ).

mera Guerra Mundial, quienes se asombraron al advertir que cuando disparaban sus cañones con un elevado ángulo de inclinación, el alcance horizontal era el doble del esperado).

\section{Conclusiones}

\subsection{Conclusiones del cálculo numérico}

- El modelo convencional, que supone que la velocidad varía sólo por la gravedad, sobreestima notablemente el alcance horizontal y también el vertical en casi todos los casos analizados. Por este motivo es inaplicable en muchos casos prácticos, tales como disparos de armas de fuego o aire comprimido, o deportes que usan pequeñas pelotas maciza: $\mathfrak{1}^{1}$ (golf, tenis, frontón), etc. Sólo es una buena aproximación a bajas velocidades $\left(v \leqslant 20 \mathrm{~m} \cdot \mathrm{s}^{-1}\right.$, no se muestra en este trabajo), o bien en tiros muy rasantes, si se admite un error relativo grande.

- Para todos los casos analizados, la fuerza más importante en los cálculos, después del peso, es la componente inercial de la resistencia del aire, dada por las fuerzas de presión. Esto sucede a velocidades moderadas o relativamente altas pero fáciles de conseguir experimentalmente.

\footnotetext{
${ }^{1}$ Las pelotas infladas suponen, generalmente, un tamaño mayor y una densidad muy baja, y están fuera del contexto de este trabajo.
}

- Debido a que la componente inercial es proporcional al cuadrado de la velocidad, su efecto es mucho mayor a velocidades altas.

- El empuje y la fuerza de rozamiento viscosa son totalmente despreciables en todos los casos analizados.

- En términos relativos, la fuerza de roce frena más a los proyectiles más pequeños y livianos.

- La fuerza de Coriolis y la variación de la densidad del aire con la altitud afectan apreciablemente las trayectorias sólo en casos de largos recorridos a grandes alturas.

- Las trayectorias numéricas presentan las siguientes características:

a) Dependen fuertemente de los parámetros considerados: ángulo de inclinación, velocidad inicial, y tamaño y densidad del proyectil.

b) Son, en general, asimétricas: la rama ascendente y la descendente tienen distintas pendientes.

c) Tienen siempre recorridos más cortos y duran menos tiempo que las trayectorias del modelo convencional.

\subsection{Discusión didáctica final}

Tanto los resultados de la encuesta como los argumentos teóricos provenientes de la epistemología, la antropología y la psicología concuerdan en que el tema de tiro oblicuo tiene un fuerte arraigo cognitivo que favorece la motivación a su estudio, como una experiencia interesante y fácil asociada a aficiones 
lúdicas. La falta de valoración de los estudiantes respecto de su valor formativo se debe, probablemente, a que ellos reparan sólo en el aspecto contextual de sus contenidos, sin advertir su valor formal, metodológico y operativo, el cual debería ser enfatizado por el docente.

La mayoría de los alumnos muestra una noción pobre y confusa respecto del efecto resistivo del aire en casos prácticos de tiro oblicuo. Sus comentarios en la encuesta, la experiencia docente y el análisis crítico de los textos de estudio indican que esta ignorancia o confusión se debe al empleo de un modelo idealizado cuyos supuestos y ámbito de validez no son debidamente analizados en la práctica. Luego, es importante que el docente aclare que el modelo idealizado supone una simplificación drástica y sólo es aplicable en casos muy específicos en que la velocidad es suficientemente baja, o bien en tiros rasantes, si se permite un error relativamente grande. En la escuela secundaria y el ciclo básico universitario, los ejemplos y los problemas prácticos deberían estar limitados a estos casos; y cabría retomar el tema, con un mayor grado de complejidad, cuando el alumno tenga los conocimientos necesarios sobre mecánica de los fluidos, en particular en las carreras científicas.

Como en todo aprendizaje de física, en tiro oblicuo es importante la experimentación. Acaso más, habida cuenta de sus bases cognitivas que participan de una dimensión lúdica. Actualmente existen diversos dispositivos accesibles - desde cañones de resorte hasta sistemas de aire comprimido de compresión graduable - que permiten lanzar pequeños proyectiles a distintas velocidades; las cuales pueden ajustarse al estudio de diferentes modelos y casos. También es posible calcular o medir, con grabadoras de video, la velocidad del proyectil en distintos tramos de su recorrido.

Luego, el estudio experimental de la resistencia del medio puede ser relativamente sencillo, pero otras fuerzas o efectos tratados en este trabajo pueden demandar mayores exigencias y dificultad. Con respecto a la variación de la densidad del aire con la altitud, el hecho de que sólo sea apreciable para proyectiles que se mantengan en un rango elevado de alturas durante una fracción considerable de su recorrido exige no sólo una considerable potencia de disparo, sino una gran disponibilidad de espacio abierto. La fuerza de Coriolis, por su parte, también supone este tipo de dificultades para poder apre- ciarse, y por tanto éste no es un tema apto, por sí solo, para ilustrar el efecto de los sistemas de referencia en rotación. En caso de que éste fuera el objetivo didáctico, debería considerarse otro tipo de experimentos (los que suelen implementarse con lanzamientos sobre plataformas giratorias, por ejemplo).

Los autores de este trabajo están actualmente diseñando prácticas de laboratorio para las carreras de física de la Universidad Nacional de Tucumán, con el fin de medir el efecto resistivo del aire y analizar la aplicabilidad de los distintos modelos en función de sus errores sistemáticos. Los resultados se comunicarán, oportunamente, en otro trabajo.

\section{Reconocimiento}

Este trabajo ha sido realizado en el marco de un proyecto de investigación subsidiado por la Secretaría de Ciencia, Arte e Innovación Tecnológica de la Universidad Nacional de Tucumán (Scait-UNT).

\section{Referencias}

[1] S. Calderón, S. González y S. Gil, Revista de Enseñanza de la Física 20, 11 (2007).

[2] S. Roncero y F. Jiménez, Aeronaves y Vehículos Espaciales, Curso de Aerodinámica de la Escuela Superior de Ingenieros de la Universidad de Sevilla, 2009, disponible en http://aero.us.es/AVE/

[3] H. Rishbeth y O. Garriot, en: Introduction to Ionospheric Physics (Academic Press Inc. Ltd, Londres, 1969), p. 5-7.

[4] R. Feynman, R. Leighton y M. Sands, en: Feynman Lectures on Physics (Addison-Wesley Publish. Co. Inc., Reading, 1964), v. 2, p. 41.5-41.9.

[5] Y. Perelman, Fúsica Recreativa I (Ed. Mir, Moscú, 1936), p. 53-56. 